\title{
Convenient Synthesis of 4-Trifluoromethyl-Substituted Imidazole Derivatives
}

\author{
Masami Kawase, ${ }^{* a}$ Setsuo Saito, ${ }^{a}$ and Teruo Kurihara ${ }^{b}$ \\ Faculty of Pharmaceutical Sciences, Josai University, ${ }^{a}$ 1-1 Keyakidai, Sakado, Saitama 350-0295, Japan and Faculty of \\ Science, Josai University, ${ }^{b} 1-1$ Keyakidai, Sakado, Saitama 350-0295, Japan. \\ Received November 29, 2000; accepted February 3, 2001
}

Mesoionic 4-trifluoroacetyl-1,3-oxazolium-5-olates (1), obtained from the reaction of $N$-acyl- $N$-alkylglycines (2) with trifluoroacetic anhydride, react with ammonia to give 4-trifluoromethyl-3,4-dihydroimidazoles (3) in high yields. Dehydration of 3 gives 4-trifluoromethylimidazoles (4) in high yields. The novel ring transformation of 1 into 3 occurs via a regioselective attack of ammonia on the C-2 position of the ring.

Key words imidazole; trifluoromethyl; 1,3-oxazolium-5-olate; ring transformation; trifluoroacetic anhydride

The synthesis of trifluoromethyl $\left(\mathrm{CF}_{3}\right)$-substituted heteroaromatic compounds has received considerable interest since many of them sometimes exhibit unique chemical, physiological or physical properties. ${ }^{1)}$ The search for a simple and efficient access to heteroaromatic compounds with a $\mathrm{CF}_{3}$ group at a specific position is an important goal in this area. $^{2)}$

Imidazole and its derivatives are an important class of heterocycles and many naturally occurring imidazoles are known to possess biological activity. ${ }^{3)}$ The imidazole nucleus is also a major component of a variety of drugs such as angiotensin II receptor antagonists, oral antiinflammatory agents, protein kinase inhibitors and fungisides. ${ }^{4)}$ Furthermore, $\mathrm{CF}_{3}$-substituted imidazoles have found applications in the medicinal and agrochemical fields. ${ }^{5}$ )

In the course of our studies on the reactivities of mesoionic 1,3-oxazolium-5-olates, ${ }^{6}$ ) we have focused on 4trifluoroacetyl-1,3-oxazolium-5-olates (1) as useful synthons for $\mathrm{CF}_{3}$-substituted heterocycles. ${ }^{7)}$

Compound 1 could easily be prepared from $\mathrm{N}$-acyl- $\mathrm{N}$ alkylglycines $\mathbf{2}$ with trifluoroacetic anhydride (TFAA). ${ }^{8)}$ In general, the mesoionic 1,3-oxazolium-5-olates are too unstable to be isolated and are utilized in situ. ${ }^{6}$ However, compound 1 with a 4-trifluoroacetyl group can be isolated and stored for several months. It is reported that compound $\mathbf{1}$ showed high reactivity towards nucleophilic reagents such as $\mathrm{H}_{2} \mathrm{O}$, EtOH, and $\mathrm{AcOH}$, bringing about ring-opening reactions. ${ }^{8)}$ These reactions occur via an initial attack of a nucleophile on the $\mathrm{C}-5$ position of the ring. ${ }^{7 \mathrm{~d})}$ We have examined the reactions of $\mathbf{1}$ with $N$-nucleophiles such as amidines, ${ }^{7 a}$ ) ammonia, ${ }^{7 b)}$ hydrazines, ${ }^{7 c)}$ and aminomalonate. ${ }^{7 e}$ In principle, nucleophilic reagents can be expected to be added to one of the three electrophilic centers at C-2, C-5, and $\mathrm{COCF}_{3}$ in 1.

Combinatorial chemistry has emerged as a powerful tool for accelerating the synthesis and screening of diverse collections of molecules. ${ }^{9,10)}$ The 1,3-dipolar cycloaddition of polymer-bound munchnones with a variety of alkynes has been developed to produce a library of up to $10^{8}$ different pyrroles. ${ }^{11)}$ The solid-phase synthesis of non-fluorinated imidazoles is reported to utilize a munchnone cycloaddition to aryltosylimines. ${ }^{12)}$ Recently, the reaction of $\mathbf{1}$ with amidines $^{7 a)}$ has also been applied to a combinatorial solidphase synthesis to prepare a library of two hundred 5-trifluo- roacetylimidazoles by Hamper et al. ${ }^{13)}$ Thus, munchnone chemistries are suitable to develop new synthesis in combinatorial chemistry.

We now present a full account of the reactions of $\mathbf{1}$ with ammonia $^{7 b)}$ and a new synthesis of 4-trifluoromethylimidazoles (4).

\section{Results and Discussion}

The 4-trifluoroacetyl-1,3-oxazolium-5-olates (1a-d) required for this study were prepared by the reaction of $N$-acyl$N$-alkylglycines $(\mathbf{2 a - d})$ with TFAA. The derivatives employed in this study, along with the products $(3 \mathbf{a}-\mathbf{i}$ and $\mathbf{4 a -}$ i), are summarized in Chart 1 . Table 1 shows the results when 4-trifluoroacetyl-1,3-oxazolium-5-olate (1a) was allowed to react with ammonium acetate under various conditions. The best result was obtained by the reaction of 1a (1 mmol) with ammonium acetate $(1.5 \mathrm{mmol})$ in $\mathrm{N}, \mathrm{N}$-dimethylformamide (DMF) $(5 \mathrm{ml})$ at $70^{\circ} \mathrm{C}$ for $2 \mathrm{~h}$ : 4-trifluoromethyldihydroimidazole (3a) was isolated in $92 \%$ yield (Table 1, run 1). Various substituted 4-trifluoroacetyl-1,3-oxazolium-5-olates $(\mathbf{1} \mathbf{a}, \mathbf{b}, \mathbf{d}, \mathbf{e})$ were subjected to a reaction under the optimum conditions, and the results are listed in Table 2. In all reactions examined, 4,5-dihydroimidazoles $(\mathbf{3 a}-\mathbf{i})$ were obtained in high yields.

A one-pot conversion of $\mathbf{2}$ to $\mathbf{3}$ also proceeded successfully. Thus, $\mathbf{2 a}$ reacted with TFAA in $\mathrm{CH}_{2} \mathrm{Cl}_{2}$ to give $\mathbf{1 a}$, which was directly subjected to the ring transformation reaction to yield $\mathbf{3 a}$ in $94 \%$ yield (Table 2, run 2). The one-pot procedure gave slightly higher yield. Pentafluoropropionic and heptafluorobutyric anhydride also reacted readily with $N$ benzoyl- $N$-methylglycine (2a) to yield 4-pentafluoroethyl(3h) and 4-heptafluoropropyldihydroimidazole (3i) by the one-pot procedure in high yields, respectively (Chart 1 and Table 2, runs 10 and 11).

The structures of $\mathbf{3 a -} \mathbf{f}$ are supported by spectral and analytical data. In the ${ }^{13} \mathrm{C}-\mathrm{NMR}$ spectra of $\mathbf{3 a}$, dihydroimidazole ring carbon atoms C2, C4 and C5 appear at 169.1, 94.01 (quartet, ${ }^{2} J_{\mathrm{C}-\mathrm{F}}=31 \mathrm{~Hz}$ ) and $59.73 \mathrm{ppm}$, respectively. The carbon of the $\mathrm{CF}_{3}$ group in $\mathbf{3 a}-\mathbf{f}$ appears at around $\delta 124 \mathrm{ppm}$ (quartet, ${ }^{1} J_{\mathrm{C}-\mathrm{F}}=285-290 \mathrm{~Hz}$ ).

Dehydration of $\mathbf{3 a}$ with $\mathrm{POCl}_{3}$ and pyridine ${ }^{14)}$ gave the known $^{15)}$ 4-trifluoromethyl-1-methyl-2-phenylimidazole (4a) in $88 \%$ yield. As shown in Table 2, various dihydroimidazoles $(\mathbf{3 a}-\mathbf{i})$ were transformed to the 4-trifluoromethylimi- 


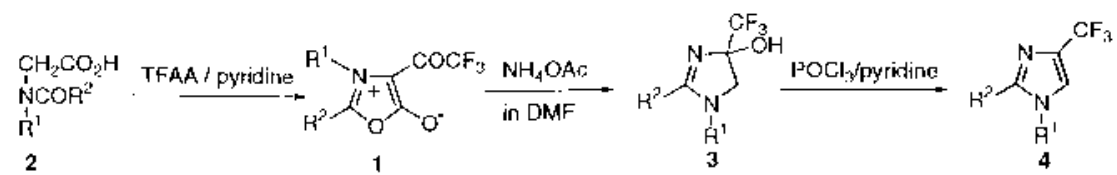

\begin{tabular}{ccc} 
1, 2,3,4 & $\mathrm{F}^{1}$ & $\mathrm{~F}^{2}$ \\
\hline $\mathbf{a}$ & $\mathrm{Me}$ & $\mathrm{Ph}^{\prime}$ \\
$\mathbf{b}$ & $\mathrm{Me}$ & $\mathrm{Bu}^{\prime}$ \\
$\mathbf{c}$ & $\mathrm{Me}$ & $\mathrm{Bn}$ \\
$\mathbf{d}$ & $\mathrm{Ph}$ & $\mathrm{Me}$ \\
$\mathbf{e}$ & $\mathrm{Ph}$ & $\mathrm{Ph}$ \\
$\mathrm{f}$ & $\mathrm{Bn}$ & $\mathrm{Ph}$ \\
$\mathrm{g}$ & $\mathrm{Bn}$ & $\mathrm{Me}$
\end{tabular}

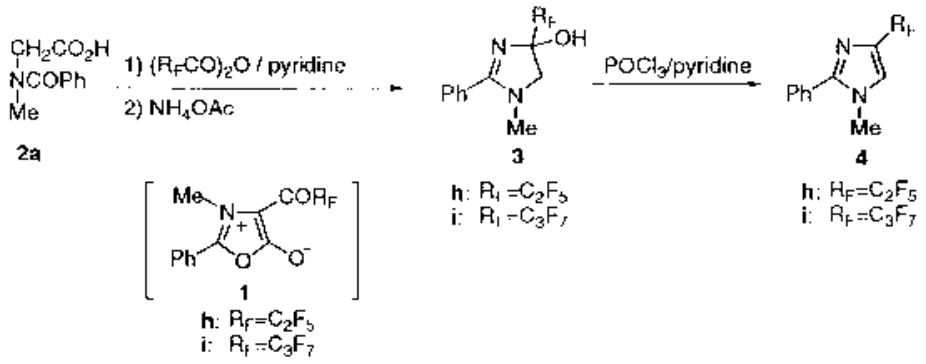

Chart 1

Table 1. Reactions of 1a with Ammonium Acetate in $\mathrm{DMF}^{a}$ )

\begin{tabular}{cclcc}
\hline \hline Run & $\mathrm{NH}_{4} \mathrm{OAc}(\mathrm{eq})^{b)}$ & Base $(\mathrm{eq})^{b)}$ & Conditions & Yield of 3a (\%) \\
\hline 1 & 1.5 & None & $70^{\circ} \mathrm{C}, 2 \mathrm{~h}$ & 92 \\
2 & 1.5 & None & $25^{\circ} \mathrm{C}, 3 \mathrm{~h}$ & 45 \\
3 & 1.5 & $\mathrm{~K}_{2} \mathrm{CO}_{3}(1.5)$ & $70^{\circ} \mathrm{C}, 2 \mathrm{~h}$ & 92 \\
4 & 3 & None & $70^{\circ} \mathrm{C}, 2 \mathrm{~h}$ & 91 \\
$5^{c)}$ & 1.5 & None & $70^{\circ} \mathrm{C}, 2 \mathrm{~h}$ & 94
\end{tabular}

a) The reactions were carried out on a $1 \mathrm{mmol}$ scale. b) Eq refers to molar equivalents with respect to 1a. c) Yield refers to the one-pot procedure using $\mathbf{2 a}$.

Table 2. Yields of 4-Trifluoromethyl-3,4-dihydroimidazoles (3) and 4-Trifluoromethylimidazoles (4)

\begin{tabular}{|c|c|c|c|}
\hline \multirow{2}{*}{ Run } & \multirow{2}{*}{ Starting materials } & \multicolumn{2}{|c|}{ Yields of products $(\%)$} \\
\hline & & 3 & 4 \\
\hline 1 & $1 \mathbf{a}$ & $\mathbf{3 a}(92)$ & $\mathbf{4 a}(88)$ \\
\hline 2 & $2 \mathbf{a}^{a)}$ & 3a (94) & - \\
\hline 3 & $1 b$ & $\mathbf{3 b}(78)$ & $\mathbf{4 b}(88)$ \\
\hline 4 & $2 c^{a)}$ & 3c (98) & $4 c(91)$ \\
\hline 5 & 1d & 3d (91) & 4d (96) \\
\hline 6 & $1 \mathrm{e}$ & $3 e(84)$ & $4 e(93)$ \\
\hline 7 & $2 \mathrm{e}^{a)}$ & 3e (91) & - \\
\hline 8 & $2 \mathbf{f}^{a)}$ & 3f (89) & 4f (99) \\
\hline 9 & $2 \mathbf{g}^{a)}$ & $3 g(85)$ & $4 g(94)$ \\
\hline $10^{b)}$ & $2 \mathbf{a}^{a)}$ & 3h (93) & 4h (99) \\
\hline $11^{c)}$ & $2 \mathbf{a}^{a)}$ & $3 \mathbf{i}(87)$ & 4i (93) \\
\hline
\end{tabular}

a) Yields refer to the one-pot procedure. b) Pentafluoropropionic anhydride was used instead of TFAA in a one-pot procedure. c) Heptafluorobutyric anhydride was used instead of TFAA in a one-pot procedure.

dazole derivatives $(\mathbf{4 a}-\mathbf{i})$ under the same conditions ${ }^{14)}$ in high yields. In the ${ }^{13} \mathrm{C}-\mathrm{NMR}$ spectra of $\mathbf{4 a}$, imidazole ring carbon atoms $\mathrm{C} 2, \mathrm{C} 4$ and $\mathrm{C} 5$ appear at 149.06, 131.56 (quartet, ${ }^{2} J_{\mathrm{C}-\mathrm{F}}=39 \mathrm{~Hz}$ ) and 121.4 (quartet, $\left.{ }^{3} J_{\mathrm{C}-\mathrm{F}}=4.1 \mathrm{~Hz}\right) \mathrm{ppm}$, respectively.

The novel ring transformation of $\mathbf{1}$ into imidazoles $\mathbf{3}$ may proceed through an initial attack of ammonia on the C-2 position of the ring. The regioselective attack of ammonia on the $\mathrm{C}-2$ position of the ring is reported to be explained in terms of the HSAB principle. ${ }^{7 d)}$ The possible mechanism has been described in ref. $7 b$.

In summary, we devised an easy method of accessing 4-trifluoromethylimidazole derivatives which are otherwise difficult to obtain. The principal advantage of using mesoionic oxazoles $\mathbf{1}$ is the great variety of substituents available for $\mathrm{R}^{1}$ and $\mathrm{R}^{2}$. This flexibility in the type of substituents in the mesoionic ring (1) will be reflected in the corresponding substitution of the resulting imidazole (4). Furthermore, the simplicity of the method and the ready availability of the starting material make this a practical approach. In addition, 4-perfluoroalkyl-substituted imidazoles could be prepared conveniently by employing perfluorocarboxylic anhydride.

\section{Experimental}

General Methods All melting points were determined using a Yanagimoto hot-stage melting point apparatus and are uncorrected. ${ }^{1} \mathrm{H}-\mathrm{NMR}$ spectra were measured on either a JEOL JNM-PMX60SI or JNM-GSX500 spectrometer with tetramethylsilane $\left(\mathrm{Me}_{4} \mathrm{Si}\right)$ as an internal reference and $\mathrm{CDCl}_{3}$ as the solvent. ${ }^{13} \mathrm{C}$-NMR spectra were obtained on a JEOL JNM-GSX500 spectrometer (at $127 \mathrm{MHz}$ ). Both ${ }^{1} \mathrm{H}$ - and ${ }^{13} \mathrm{C}-\mathrm{NMR}$ spectral data are reported in parts per million $(\delta)$ relative to $\mathrm{Me}_{4} \mathrm{Si}$. Infrared (IR) spectra were recorded on a JASCO IR810 spectrometer. Low- and high-resolution MS were obtained with a JEOL JMS-DX300 spectrometer with a direct inlet system at $70 \mathrm{eV}$. Elemental analyses were carried out in the microanalytical laboratory of this university. Standard workup means that the organic layers were finally dried over $\mathrm{Na}_{2} \mathrm{SO}_{4}$, filtered, and concentrated in vacuo below 45 ${ }^{\circ} \mathrm{C}$ using a rotary evaporator.

Materials The following compounds were prepared by reported procedures: $N$-Benzoyl- $N$-methylglycine (2a): $\mathrm{mp} 101-104^{\circ} \mathrm{C}\left(\mathrm{mp}^{16)} 102-104\right.$ $\left.{ }^{\circ} \mathrm{C}\right) . N$-Methyl- $N$-pivaloylglycine (2b): $\left.\mathrm{mp} 75-76^{\circ} \mathrm{C}\left(\mathrm{mp}^{7 f}\right) 75-76^{\circ} \mathrm{C}\right) . \mathrm{N}$ Acetyl- $N$-phenylglycine $(\mathbf{2 d}): \mathrm{mp} \quad 196-198^{\circ} \mathrm{C}\left(\mathrm{mp}^{16)} 193-195^{\circ} \mathrm{C}\right) . \mathrm{N}$ Benzoyl- $N$-phenylglycine (2e): mp $190-193{ }^{\circ} \mathrm{C}\left(\mathrm{mp}^{16)} 193-195^{\circ} \mathrm{C}\right)$. Benzyl $N$-phenylacetyl- $N$-methylglycinate $(\mathbf{5 a})$ : Yield $89 \%$. bp 5 , $228-230^{\circ} \mathrm{C}$. ${ }^{1} \mathrm{H}-\mathrm{NMR}(60 \mathrm{MHz}) \delta: 3.00+3.03(\mathrm{~s}, 3 \mathrm{H}), 3.67+3.77(\mathrm{~s}, 2 \mathrm{H}), 4.03+4.20(\mathrm{~s}$, $2 \mathrm{H}), 5.10+5.17(\mathrm{~s}, 2 \mathrm{H}), 7.27+7.33(\mathrm{~s}, 10 \mathrm{H})$. IR (Nujol) $\mathrm{cm}^{-1}: 1650,1740$. MS $m / z$ : $297\left(\mathrm{M}^{+}, 11 \%\right), 91(100 \%)$. High-resolution MS: Calcd for $\mathrm{C}_{18} \mathrm{H}_{19} \mathrm{NO}_{3}: 297.1365$. Found: 297.1367 . Ethyl $N$-benzoyl $-N$-benzylglycinate (5b): Yield 94\%. Oil. IR (Nujol) $\mathrm{cm}^{-1}: 1635,1740 .{ }^{1} \mathrm{H}-\mathrm{NMR}(500 \mathrm{MHz}) \delta$ : $1.21-1.24+1.25-1.30(\mathrm{~m}, 3 \mathrm{H}), 3.85+4.15(\mathrm{~s}, 2 \mathrm{H}), 4.00-4.17+4.20-$ $4.24(\mathrm{~m}, 2 \mathrm{H}), 4.62+4.82(\mathrm{~s}, 2 \mathrm{H}), 7.20-7.53(\mathrm{~m}, 10 \mathrm{H}) . \mathrm{MS} m / z: 297\left(\mathrm{M}^{+}\right.$, $3 \%), 192$ (100\%). High-resolution MS: Calcd for $\mathrm{C}_{18} \mathrm{H}_{19} \mathrm{NO}_{3}$ : 297.1365 . 
Found: 297.1360. Ethyl $N$-acetyl- $N$-benzylglycinate (5c): Yield 91\%. Oil. IR (Nujol) $\mathrm{cm}^{-1}: 1650,1740 .{ }^{1} \mathrm{H}-\mathrm{NMR}(500 \mathrm{MHz}) \delta: 1.22-1.28(\mathrm{~m}, 3 \mathrm{H})$, $2.13+2.22(\mathrm{~s}, 3 \mathrm{H}), 3.91+4.05(\mathrm{~s}, 2 \mathrm{H}), 4.14-4.20(\mathrm{~m}, 2 \mathrm{H}), 4.63-4.65(\mathrm{~s}$, 2H), $7.19-7.38(\mathrm{~m}, 5 \mathrm{H})$. MS $m / z: 235\left(\mathrm{M}^{+}, 4 \%\right), 192(100 \%)$. High-resolution MS: Calcd for $\mathrm{C}_{13} \mathrm{H}_{17} \mathrm{NO}_{3}: 235.1209$. Found: 235.1225 .

$\mathrm{N}$-Phenylacetyl- $\mathrm{N}$-methylglycine (2c) A mixture of benzyl $\mathrm{N}$-phenylacetyl- $N$-methylglycinate $(\mathbf{5 a})(7.5 \mathrm{~g}, 25 \mathrm{~mol})$ and $10 \% \mathrm{Pd}-\mathrm{C}(300 \mathrm{mg})$ in AcOEt $(70 \mathrm{ml})$ was stirred under a $\mathrm{H}_{2}$ atmosphere at room temperature for $0.5 \mathrm{~h}$. The mixture was filtered and the filtrate was concentrated in vacuo. The residue was purified by column chromatography on silica gel with $\mathrm{CH}_{2} \mathrm{Cl}_{2}-\mathrm{MeOH}(10: 1)$ to give $2 \mathrm{c}(4.9 \mathrm{~g}, 94 \%)$ as a colorless oil. ${ }^{1} \mathrm{H}-\mathrm{NMR}$ $(60 \mathrm{MHz}) \delta: 2.97+3.00(\mathrm{~s}, 3 \mathrm{H}), 3.63+3.77(\mathrm{~s}, 2 \mathrm{H}), 3.97+4.10(\mathrm{~s}, 2 \mathrm{H}), 7.20$ (br s, 5H). 10.00 (s, 1H). IR (Nujol) $\mathrm{cm}^{-1}: 1640,1740,2200-3600$ (br). MS $m / z$ : $207\left(\mathrm{M}^{+}, 12 \%\right), 91(100 \%)$. High-resolution MS: Calcd for $\mathrm{C}_{11} \mathrm{H}_{13} \mathrm{NO}_{3}: 207.0895$. Found: 207.0874 .

Hydrolysis of Ethyl $N$-Benzoyl- $N$-benzylglycinate (5b) and Ethyl $N$ Acetyl- $N$-benzylglycinate (5c) A mixture of ethyl ester $(\mathbf{5 b}, \mathbf{c})(14 \mathrm{mmol})$ and $2 \mathrm{~N} \mathrm{NaOH}(9.3 \mathrm{ml}, 18 \mathrm{mmol})$ in dioxane $(9.3 \mathrm{ml})$ was stirred at $65^{\circ} \mathrm{C}$ for $3 \mathrm{~h}$. The reaction mixture was diluted with $\mathrm{Et}_{2} \mathrm{O}(100 \mathrm{ml})$ and $\mathrm{H}_{2} \mathrm{O}(100 \mathrm{ml})$. The aqueous layer was acidified with conc. $\mathrm{HCl}$ and extracted with $\mathrm{AcOEt}$ $(100 \mathrm{ml} \times 2)$ followed by standard workup to give the desired acids $(\mathbf{2 f}$ and $\mathbf{g})$ in high yields.

$N$-Benzoyl- $N$-benzylglycine (2f): mp $106-107^{\circ} \mathrm{C}$ (AcOEt-hexane). IR (Nujol) $\mathrm{cm}^{-1}: 1610,1740,2500-3300$ (br). ${ }^{1} \mathrm{H}-\mathrm{NMR}(500 \mathrm{MHz}) \delta$ : $3.86+4.19(\mathrm{~s}, 2 \mathrm{H}), 4.63+4.81(\mathrm{~s}, 2 \mathrm{H}), 6.62-6.92(\mathrm{br}, 1 \mathrm{H}), 7.20-7.54(\mathrm{~m}$, $10 \mathrm{H})$. MS m/z: $269\left(\mathrm{M}^{+}, 24 \%\right), 105(100 \%)$. Anal. Calcd for $\mathrm{C}_{16} \mathrm{H}_{15} \mathrm{NO}_{3}: \mathrm{C}$, 71.36; H, 5.61; N, 5.20. Found: C, 71.23; H, 5.78; N, 5.03 .

$\mathrm{N}$-Acetyl- $\mathrm{N}$-benzylglycine (2g): $\mathrm{mp} 118-119^{\circ} \mathrm{C}$ (AcOEt-hexane). IR (Nujol) $\mathrm{cm}^{-1}: 1610,1725,2200-3200$ (br). ${ }^{1} \mathrm{H}-\mathrm{NMR}(500 \mathrm{MHz}) \delta: 2.16+$ $2.24(\mathrm{~s}, 3 \mathrm{H}), 3.94+4.08(\mathrm{~s}, 2 \mathrm{H}), 4.63+4.66(\mathrm{~s}, 2 \mathrm{H}), 7.18-7.40(\mathrm{~m}, 5 \mathrm{H})$, 7.48-7.64 (br, 1H). MS m/z: $207\left(\mathrm{M}^{+}, 13 \%\right), 164(100 \%)$. Anal. Calcd for $\mathrm{C}_{11} \mathrm{H}_{13} \mathrm{NO}_{3}$ : C, 63.76; H, 6.32; N, 6.76. Found: C, 63.60; H, 6.32; N, 6.60.

General Procedure for the Preparation of 4-Trifluoroacetyl-1,3-oxazolium-5-olates (1) TFAA $(11 \mathrm{ml}, 78 \mathrm{mmol})$ was added to a stirred solution of $N$-acyl- $N$-alkylglycine $(26 \mathrm{mmol})$ in $\mathrm{CH}_{2} \mathrm{Cl}_{2}(50 \mathrm{ml})$ at $0{ }^{\circ} \mathrm{C}$ for 10 min. The mixture was stirred at $25^{\circ} \mathrm{C}$ for $3 \mathrm{~h}$ and then extracted with $\mathrm{CH}_{2} \mathrm{Cl}_{2}$ $(80 \mathrm{ml} \times 2)$. The combined extracts were washed successively with $3 \% \mathrm{HCl}$, $\mathrm{H}_{2} \mathrm{O}, 1 \% \mathrm{Na}_{2} \mathrm{CO}_{3}$, and $\mathrm{H}_{2} \mathrm{O}$. After the standard workup, the residue was recrystallized from $\mathrm{CH}_{2} \mathrm{Cl}_{2}$-hexane to give the 4-trifluoroacetyl-1,3-oxazolium-5-olates (1).

3-Methyl-2-phenyl-4-trifluoroacetyl-1,3-oxazolium-5-olate (1a): Yield $92 \%$. mp $161-163^{\circ} \mathrm{C}\left(\mathrm{mp}^{8 b)} 162-163^{\circ} \mathrm{C}\right) .2$-tert-Butyl-3-methyl-4-trifluoroacetyl-1,3-oxazolium-5-olate (1b): Yield $94 \%$. mp $120-121^{\circ} \mathrm{C}\left(\mathrm{mp}^{7 f}\right)$ 120-121 ${ }^{\circ} \mathrm{C}$ ). 2-Methyl-3-phenyl-4-trifluoroacetyl-1,3-oxazolium-5-olate (1d): Yield $78 \%$. mp $200-203^{\circ} \mathrm{C}\left(\mathrm{mp}^{8 b)} 211-212^{\circ} \mathrm{C}\right)$. 2,3-Diphenyl-4-trifluoroacetyl-1,3-oxazolium-5-olate (1e): Yield $81 \%$. mp 194-196 ${ }^{\circ} \mathrm{C}\left(\mathrm{mp}^{8 b}\right)$ $194-196^{\circ} \mathrm{C}$ )

General Procedure for the Reactions of 4-Trifluoroacetyl-1,3-oxazolium-5-olates (1) with Ammonium Acetate A solution of $\mathbf{1}(1.5 \mathrm{mmol})$ and ammonium acetate $(2.2 \mathrm{mmol})$ in dry DMF $(5 \mathrm{ml})$ was stirred at $70{ }^{\circ} \mathrm{C}$ for $2 \mathrm{~h}$. The mixture was diluted with AcOEt $(40 \mathrm{ml})$ and $\mathrm{H}_{2} \mathrm{O}(30 \mathrm{ml})$. After the standard workup, the residue was recrystallized from EtOAc-hexane to give the product $(\mathbf{3})$.

The following products were identified by comparison of the IR and ${ }^{1} \mathrm{H}-$ NMR spectral data with those of authentic samples. ${ }^{7 f)}$ 4-Trifluoromethyl-4hydroxy-1-methyl-2-phenyl-4,5-dihydroimidazole (3a): mp $196-198^{\circ} \mathrm{C}$ $\left(\mathrm{Et}_{2} \mathrm{O}\right)$. 2-tert-Butyl-4-trifluoromethyl-4-hydroxy-1-methyl-4,5-dihydroimidazole (3b): $\mathrm{mp} 155-157^{\circ} \mathrm{C} \quad\left(\mathrm{Et}_{2} \mathrm{O}\right)$. 4-Trifluoromethyl-4-hydroxy-2methyl-1-phenyl-4,5-dihydroimidazole (3d): $\mathrm{mp} 201-202^{\circ} \mathrm{C}\left(\mathrm{Et}_{2} \mathrm{O}\right)$. 4-Trifluoromethyl-4-hydroxy-1,2-diphenyl-4,5-dihydroimidazole (3e): $\mathrm{mp} \mathrm{174-}$ $175^{\circ} \mathrm{C}\left(\mathrm{Et}_{2} \mathrm{O}\right)$.

One-Pot Procedure for the Conversion of $\mathrm{N}$-Acyl- $\mathrm{N}$-alkylglycine (2) to Dihydroimidazolines (3) TFAA $(0.64 \mathrm{ml}, 4.5 \mathrm{mmol})$ was added to a stirred solution of $\mathrm{N}$-acyl- $\mathrm{N}$-alkylglycine (2) $(1.5 \mathrm{mmol})$ in dry $\mathrm{CH}_{2} \mathrm{Cl}_{2}(4$ $\mathrm{ml}$ ) at $0{ }^{\circ} \mathrm{C}$. The mixture was stirred at $25^{\circ} \mathrm{C}$ for $3 \mathrm{~h}$ and the solvents were evaporated to dryness. The residue was dissolved in dry DMF $(6 \mathrm{ml})$ and ammonium acetate $(182 \mathrm{mg}, 2.2 \mathrm{mmol})$ was added to the DMF solution at 0 ${ }^{\circ} \mathrm{C}$. The mixture was stirred at $70{ }^{\circ} \mathrm{C}$ for $2 \mathrm{~h}$. After the standard workup, the residue was recrystallized from EtOAc-hexane to give the product (3).

2-Benzyl-4-trifluoromethyl-4-hydroxy-1-methyl-4,5-dihydroimidazole (3c): 3c; mp $176-177^{\circ} \mathrm{C}\left(\mathrm{Et}_{2} \mathrm{O}\right)$. IR (Nujol) $\mathrm{cm}^{-1}: 2500-3600$ (br), 1575. ${ }^{1} \mathrm{H}-\mathrm{NMR}(500 \mathrm{MHz}) \delta: 2.75(\mathrm{~s}, 3 \mathrm{H}), 3.46(\mathrm{~d}, 1 \mathrm{H}, J=11.0 \mathrm{~Hz}), 3.61(\mathrm{~d}, 1 \mathrm{H}$, $J=15.3 \mathrm{~Hz}), 3.62(\mathrm{~d}, 1 \mathrm{H}, J=11.0 \mathrm{~Hz}), 3.82(\mathrm{~d}, 1 \mathrm{H}, J=15.3 \mathrm{~Hz}), 7.24(\mathrm{t}, 1 \mathrm{H}$, $J=7.3 \mathrm{~Hz}), 7.25$ (d, $2 \mathrm{H}, J=7.3 \mathrm{~Hz}), 7.30$ (t, $2 \mathrm{H}, J=7.3 \mathrm{~Hz}) \cdot{ }^{13} \mathrm{C}-\mathrm{NMR}(127$
MHz) $\delta: 32.43\left(\mathrm{CH}_{2}\right), 33.73\left(\mathrm{CH}_{2}\right), 59.22\left(\mathrm{CH}_{3}\right), 93.66\left(\mathrm{C},{ }^{2} J_{\mathrm{C}-\mathrm{F}}=32 \mathrm{~Hz}\right)$, $124.42\left(\mathrm{CF}_{3},{ }^{1} J_{\mathrm{C}-\mathrm{F}}=285 \mathrm{~Hz}\right), 127.08(\mathrm{CH}), 128.27(\mathrm{CH}), 128.84(\mathrm{CH})$, 134.23 (C), 168.44 (C). MS m/z: $258\left(\mathrm{M}^{+}, 8 \%\right), 91(100 \%)$. Anal. Calcd for $\mathrm{C}_{12} \mathrm{H}_{13} \mathrm{~F}_{3} \mathrm{~N}_{2} \mathrm{O}: \mathrm{C}, 55.81 ; \mathrm{H}, 5.07 ; \mathrm{N}, 10.85$. Found: $\mathrm{C}, 55.83 ; \mathrm{H}, 5.10 ; \mathrm{N}$, 10.90 .

1-Benzyl-4-trifluoromethyl-4-hydroxy-2-phenyl-4,5-dihydroimidazole (3f): 3f; $\mathrm{mp} 175-177^{\circ} \mathrm{C}\left(\mathrm{CH}_{2} \mathrm{Cl}_{2}\right.$-hexane). IR (Nujol) $\mathrm{cm}^{-1}: 2550-3600$ (br), 1575. ${ }^{1} \mathrm{H}-\mathrm{NMR}(500 \mathrm{MHz}) \delta: 3.50(\mathrm{~d}, 1 \mathrm{H}, J=11.8 \mathrm{~Hz}), 3.67(\mathrm{~d}, 1 \mathrm{H}$, $J=11.8 \mathrm{~Hz}), 4.41(\mathrm{~d}, 1 \mathrm{H}, J=16.0 \mathrm{~Hz}), 4.46(\mathrm{~d}, 1 \mathrm{H}, J=16.0 \mathrm{~Hz}), 7.02-7.15$ (br, $1 \mathrm{H}), 7.18(\mathrm{~d}, 2 \mathrm{H}, J=7.9 \mathrm{~Hz}), 7.30(\mathrm{t}, 1 \mathrm{H}, J=7.3 \mathrm{~Hz}), 7.36(\mathrm{t}, 2 \mathrm{H}, J=7.3$ $\mathrm{Hz}), 7.43(\mathrm{t}, 2 \mathrm{H}, J=7.9 \mathrm{~Hz}), 7.48(\mathrm{t}, 1 \mathrm{H}, J=7.3 \mathrm{~Hz}), 7.61(\mathrm{~d}, 2 \mathrm{H}, J=7.3 \mathrm{~Hz})$. ${ }^{13} \mathrm{C}-\mathrm{NMR}(127 \mathrm{MHz}) \delta: 50.32\left(\mathrm{CH}_{2}\right), 56.14\left(\mathrm{CH}_{2}\right), 93.49\left(\mathrm{C},{ }^{2} J_{\mathrm{C}-\mathrm{F}}=31 \mathrm{~Hz}\right)$, $123.51\left(\mathrm{CF}_{3},{ }^{1} J_{\mathrm{C}-\mathrm{F}}=284 \mathrm{~Hz}\right), 125.94(\mathrm{CH}), 126.84(\mathrm{CH}), 127.51(\mathrm{CH})$, $127.78(\mathrm{CH}), 128.02(\mathrm{CH}), 128.54(\mathrm{C}), 129.99(\mathrm{CH}), 135.53(\mathrm{C}), 168.41$ (C). MS m/z: $320\left(\mathrm{M}^{+}, 1 \%\right), 91(100 \%)$. Anal. Calcd for $\mathrm{C}_{17} \mathrm{H}_{15} \mathrm{~F}_{3} \mathrm{~N}_{2} \mathrm{O}: \mathrm{C}$, $63.75 ; \mathrm{H}, 4.72 ; \mathrm{N}, 8.75$. Found: C, 63.40; H, 4.57; N, 8.69.

1-Benzyl-4-trifluoromethyl-4-hydroxy-2-methyl-4,5-dihydroimidazole (3g): 3g; mp $155-156{ }^{\circ} \mathrm{C}\left(\mathrm{CH}_{2} \mathrm{Cl}_{2}-\right.$ hexane). IR (Nujol) $\mathrm{cm}^{-1}: 2500-3600$ (br), 1585. ${ }^{1} \mathrm{H}-\mathrm{NMR}(500 \mathrm{MHz}) \delta: 2.00(\mathrm{~s}, 3 \mathrm{H}), 3.33(\mathrm{~d}, 1 \mathrm{H}, J=10.8 \mathrm{~Hz})$, $3.53(\mathrm{~d}, 1 \mathrm{H}, J=10.8 \mathrm{~Hz}), 4.34(\mathrm{~d}, 1 \mathrm{H}, J=16.0 \mathrm{~Hz}), 4.42(\mathrm{~d}, 1 \mathrm{H}, J=16.0 \mathrm{~Hz})$, $7.09(\mathrm{~d}, 2 \mathrm{H}, J=7.6 \mathrm{~Hz}), 7.24(\mathrm{t}, 1 \mathrm{H}, J=7.6 \mathrm{~Hz}), 7.31(\mathrm{t}, 2 \mathrm{H}, J=7.6 \mathrm{~Hz}) \cdot{ }^{13} \mathrm{C}-$ $\operatorname{NMR}(127 \mathrm{MHz}) \delta: 13.39\left(\mathrm{CH}_{3}\right), 49.10\left(\mathrm{CH}_{2}\right), 56.48\left(\mathrm{CH}_{2}\right), 93.50\left(\mathrm{C},{ }^{2} J_{\mathrm{C}-\mathrm{F}}=\right.$ $31 \mathrm{~Hz}), 124.39\left(\mathrm{CF}_{3},{ }^{1} J_{\mathrm{C}-\mathrm{F}}=284 \mathrm{~Hz}\right), 126.52(\mathrm{CH}), 127.83(\mathrm{CH}), 129.03$ (CH), 135.76 (C), 167.14 (C). MS m/z: $258\left(\mathrm{M}^{+}, 5 \%\right), 91$ (100\%). Anal. Calcd for $\mathrm{C}_{12} \mathrm{H}_{13} \mathrm{~F}_{3} \mathrm{~N}_{2} \mathrm{O}: \mathrm{C}, 55.81 ; \mathrm{H}, 5.07 ; \mathrm{N}, 10.85$. Found: $\mathrm{C}, 55.85 ; \mathrm{H}$, $5.02 ; \mathrm{N}, 10.72$

The following products were identified by comparison of the IR and ${ }^{1} \mathrm{H}-$ NMR spectral data with those of authentic samples ${ }^{7 f)}$ : 4-Pentafluoroethyl-4hydroxy-1-methyl-2-phenyl-4,5-dihydroimidazole (3h) mp $183-186{ }^{\circ} \mathrm{C}$ $\left(\mathrm{Et}_{2} \mathrm{O}\right)$. 4-Heptafluoropropyl-4-hydroxy-1-methyl-2-phenyl-4,5-dihydroimidazole (3i): $\mathrm{mp} 188-189^{\circ} \mathrm{C}\left(\mathrm{Et}_{2} \mathrm{O}\right)$.

General Procedure for the Dehydration of 3,4-Dihydroimidazoles (3) to Afford Imidazoles (4) $\mathrm{POCl}_{3}(0.23 \mathrm{ml}, 2.5 \mathrm{mmol})$ was added to a stirred solution of $3(1.0 \mathrm{mmol})$ in pyridine $(0.73 \mathrm{ml}, 9.0 \mathrm{mmol})$ at $0{ }^{\circ} \mathrm{C}$ and the mixture was heated at $90^{\circ} \mathrm{C}$ for $2 \mathrm{~h}$. The solvent was evaporated to dryness and the residue was extracted with AcOEt $(40 \mathrm{ml} \times 2)$. After the standard workup, the residue was purified by column chromatography on silica gel with EtOAc-hexane $(1: 2)$ to give the product 4.

4-Trifluoromethyl-1-methyl-2-phenylimidazole (4a): $\mathrm{mp} \quad 59-60^{\circ} \mathrm{C}$ (hexane) $\left(\mathrm{mp}^{15)} 61.5-62.5^{\circ} \mathrm{C}\right) .{ }^{1} \mathrm{H}-\mathrm{NMR}(500 \mathrm{MHz}) \delta: 3.76(3 \mathrm{H}, \mathrm{s}), 7.31$ $(1 \mathrm{H}, \mathrm{s}), 7.45-7.48(3 \mathrm{H}, \mathrm{m}), 7.61-7.63(2 \mathrm{H}, \mathrm{m}) .{ }^{13} \mathrm{C}-\mathrm{NMR}(127 \mathrm{MHz}) \delta$ : $34.82\left(\mathrm{CH}_{3}\right), 121.74\left(\mathrm{CH},{ }^{3} J_{\mathrm{C}-\mathrm{F}}=4.1 \mathrm{~Hz}\right), 121.77\left(\mathrm{CF}_{3},{ }^{1} J_{\mathrm{C}-\mathrm{F}}=267 \mathrm{~Hz}\right)$, $128.69(\mathrm{CH}), 128.95(\mathrm{CH}), 129.27(\mathrm{C}), 129.54(\mathrm{CH}), 131.56\left(\mathrm{C},{ }^{2} J_{\mathrm{C}-\mathrm{F}}=39\right.$ $\mathrm{Hz}), 149.06(\mathrm{~s})$. MS $m / z: 226\left(\mathrm{M}^{+}, 100 \%\right)$.

2-tert-Butyl-4-trifluoromethyl-1-methylimidazole (4b): $\mathbf{4 b} ; \mathrm{mp} 46-47{ }^{\circ} \mathrm{C}$ (hexane). ${ }^{1} \mathrm{H}-\mathrm{NMR}(500 \mathrm{MHz}) \delta: 1.44(\mathrm{~s}, 9 \mathrm{H}), 3.78(\mathrm{~s}, 3 \mathrm{H}), 7.09(\mathrm{q}, 1 \mathrm{H}$, $J=1.2 \mathrm{~Hz}) .{ }^{13} \mathrm{C}-\mathrm{NMR}(127 \mathrm{MHz}) \delta: 29.08\left(\mathrm{CH}_{3}\right), 33.50(\mathrm{C}), 35.77\left(\mathrm{CH}_{3}\right)$, $122.01\left(\mathrm{CF}_{3},{ }^{1} J_{\mathrm{C}-\mathrm{F}}=267 \mathrm{~Hz}\right), 122.58\left(\mathrm{CH},{ }^{3} J_{\mathrm{C}-\mathrm{F}}=4 \mathrm{~Hz}\right), 128.95\left(\mathrm{C},{ }^{2} J_{\mathrm{C}-\mathrm{F}}=38\right.$ $\mathrm{Hz}), 155.65$ (C). MS m/z: $206\left(\mathrm{M}^{+}, 27 \%\right), 191(100 \%)$. Anal. Calcd for $\mathrm{C}_{9} \mathrm{H}_{13} \mathrm{~F}_{3} \mathrm{~N}_{2}: \mathrm{C}, 52.42 ; \mathrm{H}, 6.35 ; \mathrm{N}, 13.58$. Found: $\mathrm{C}, 52.70 ; \mathrm{H}, 6.16 ; \mathrm{N}, 13.44$.

2-Benzyl-4-trifluoromethyl-1-methylimidazole (4c): $4 \mathbf{c}$; bp $145^{\circ} \mathrm{C}$ (bath temperature). ${ }^{1} \mathrm{H}-\mathrm{NMR}(500 \mathrm{MHz}) \delta: 3.43(\mathrm{~s}, 3 \mathrm{H}), 4.12(\mathrm{~s}, 2 \mathrm{H}), 7.14(\mathrm{~d}, 2 \mathrm{H}$, $J=7.9 \mathrm{~Hz}), 7.17(\mathrm{br} \mathrm{s}, 1 \mathrm{H}), 7.23(\mathrm{t}, 1 \mathrm{H}, J=7.6 \mathrm{~Hz}), 7.29(\mathrm{t}, 2 \mathrm{H}, J=7.6 \mathrm{~Hz})$. ${ }^{13} \mathrm{C}-\mathrm{NMR}(127 \mathrm{MHz}) \delta: 33.38\left(\mathrm{CH}_{2}\right), 33.61\left(\mathrm{CH}_{3}\right), 121.08\left(\mathrm{CH},{ }^{3} J_{\mathrm{C}-\mathrm{F}}=4\right.$ $\mathrm{Hz}), 121.85\left(\mathrm{CF}_{3},{ }^{1} J_{\mathrm{CF}}=267 \mathrm{~Hz}\right), 127.01(\mathrm{CH}), 128.25(\mathrm{CH}), 128.88(\mathrm{CH})$, $130.41\left(\mathrm{C},{ }^{2} J_{\mathrm{C}-\mathrm{F}}=39 \mathrm{~Hz}\right), 135.99$ (C), 148.17 (C). MS m/z: $240\left(\mathrm{M}^{+}, 87 \%\right)$, $91(100 \%)$. Anal. Calcd for $\mathrm{C}_{12} \mathrm{H}_{11} \mathrm{~F}_{3} \mathrm{~N}_{2}$ : C, 60.00; H, 4.62; N, 11.66. Found: C, 60.19; H, 4.63; N, 11.84 .

4-Trifluoromethyl-2-methyl-1-phenylimidazole (4d): 4d; mp $68-69^{\circ} \mathrm{C}$ (Et ${ }_{2} \mathrm{O}-$ hexane). ${ }^{1} \mathrm{H}-\mathrm{NMR}(500 \mathrm{MHz}) \delta: 2.37(\mathrm{~s}, 3 \mathrm{H}), 7.31$ (dd, $2 \mathrm{H}, J=1.5$, $7.9 \mathrm{~Hz}), 7.34(\mathrm{q}, 1 \mathrm{H}, J=1.2 \mathrm{~Hz}), 7.49-7.55(\mathrm{~m}, 3 \mathrm{H}) .{ }^{13} \mathrm{C}-\mathrm{NMR}(127 \mathrm{MHz})$ $\delta: 13.67\left(\mathrm{CH}_{3}\right), 120.47\left(\mathrm{CH},{ }^{3} J_{\mathrm{C}-\mathrm{F}}=4 \mathrm{~Hz}\right), 121.75\left(\mathrm{CF}_{3},{ }^{1} J_{\mathrm{C}-\mathrm{F}}=267 \mathrm{~Hz}\right)$, $125.64(\mathrm{CH}), 129.18(\mathrm{CH}), 129.83(\mathrm{CH}), 131.13\left(\mathrm{C},{ }^{2} J_{\mathrm{C}-\mathrm{F}}=38 \mathrm{~Hz}\right), 136.80$ (C), 146.35 (C). MS m/z: $226\left(\mathrm{M}^{+}, 100 \%\right)$. Anal. Calcd for $\mathrm{C}_{11} \mathrm{H}_{9} \mathrm{~F}_{3} \mathrm{~N}_{2}: \mathrm{C}$, $58.41 ; \mathrm{H}, 4.01 ; \mathrm{N}, 12.38$. Found: C, 58.22; H, 4.05; N, 12.34 .

4-Trifluoromethyl-1,2-diphenylimidazole (4e): $\quad 4 \mathrm{e} ; \quad \mathrm{mp} \quad 122-123{ }^{\circ} \mathrm{C}$ (Et ${ }_{2} \mathrm{O}$-hexane). ${ }^{1} \mathrm{H}-\mathrm{NMR}(500 \mathrm{MHz}) \delta: 7.21-7.25(\mathrm{~m}, 3 \mathrm{H}), 7.26(\mathrm{br} \mathrm{s}, 1 \mathrm{H})$, $7.29-7.33(\mathrm{~m}, 1 \mathrm{H}), 7.37-7.41(\mathrm{~m}, 2 \mathrm{H}), 7.42-7.45(\mathrm{~m}, 3 \mathrm{H}), 7.47-7.49$ $(\mathrm{m}, 1 \mathrm{H}) .{ }^{13} \mathrm{C}-\mathrm{NMR}(127 \mathrm{MHz}) \delta: 121.73\left(\mathrm{CF}_{3},{ }^{1} J_{\mathrm{C}-\mathrm{F}}=267 \mathrm{~Hz}\right), 122.24(\mathrm{CH}$, $\left.{ }^{3} J_{\mathrm{C}-\mathrm{F}}=4 \mathrm{~Hz}\right), 125.86(\mathrm{CH}), 128.32(\mathrm{CH}), 128.89(\mathrm{CH} \times 2), 129.03(\mathrm{CH})$, $129.22(\mathrm{C}), 129.77(\mathrm{CH}), 132.28\left(\mathrm{C},{ }^{2} J_{\mathrm{C}-\mathrm{F}}=39 \mathrm{~Hz}\right), 137.47(\mathrm{C}), 147.95(\mathrm{C})$. MS $m / z: 288\left(\mathrm{M}^{+}, 100 \%\right)$. Anal. Calcd for $\mathrm{C}_{16} \mathrm{H}_{11} \mathrm{~F}_{3} \mathrm{~N}_{2}: \mathrm{C}, 66.67 ; \mathrm{H}, 3.85 ; \mathrm{N}$, 9.72. Found: C, $66.87 ; \mathrm{H}, 4.08 ; \mathrm{N}, 9.62$.

1-Benzyl-4-trifluoromethyl-2-phenylimidazole (4f): $4 \mathbf{f} ; \mathrm{mp} \quad 83-84^{\circ} \mathrm{C}$ 
(Et ${ }_{2} \mathrm{O}$-hexane). ${ }^{1} \mathrm{H}-\mathrm{NMR}(500 \mathrm{MHz}) \delta: 5.20(\mathrm{~s}, 2 \mathrm{H}), 7.09(\mathrm{~d}, 2 \mathrm{H}, J=7.5$ $\mathrm{Hz}), 7.27$ (br s, $1 \mathrm{H}), 7.32-7.39(\mathrm{~m}, 3 \mathrm{H}), 7.40-7.46(\mathrm{~m}, 3 \mathrm{H}), 7.56(\mathrm{dd}, 2 \mathrm{H}$ $J=2.1,7.3 \mathrm{~Hz}) .{ }^{13} \mathrm{C}-\mathrm{NMR}(127 \mathrm{MHz}) \delta: 50.81\left(\mathrm{CH}_{2}\right), 120.60\left(\mathrm{CH},{ }^{3} J_{\mathrm{C}-\mathrm{F}}=4\right.$ $\mathrm{Hz}), 121.67\left(\mathrm{CF}_{3},{ }^{1} J_{\mathrm{C}-\mathrm{F}}=267 \mathrm{~Hz}\right), 126.81(\mathrm{CH} \times 2), 128.39(\mathrm{CH}) .128 .68$ $(\mathrm{CH}), 129.04(\mathrm{CH}), 129.16(\mathrm{CH}), 129.66(\mathrm{C}), 131.93\left(\mathrm{C},{ }^{2} J_{\mathrm{C}-\mathrm{F}}=39 \mathrm{~Hz}\right)$, 135.58 (C), 149.34 (C). MS m/z: $302\left(\mathrm{M}^{+}, 17 \%\right), 91$ (100\%). Anal. Calcd for $\mathrm{C}_{17} \mathrm{H}_{13} \mathrm{~F}_{3} \mathrm{~N}_{2}: \mathrm{C}, 67.75 ; \mathrm{H}, 4.33 ; \mathrm{N}, 9.27$. Found: $\mathrm{C}, 67.59 ; \mathrm{H}, 4.47 ; \mathrm{N}$, 9.30 .

1-Benzyl-4-trifluoromethyl-2-methylimidazole $(\mathbf{4 g}): \mathbf{4 g} ; \mathrm{mp} \quad 79-80^{\circ} \mathrm{C}$ (Et $\mathrm{E}_{2} \mathrm{O}$-hexane). ${ }^{1} \mathrm{H}-\mathrm{NMR}(500 \mathrm{MHz}) \delta: 2.33(\mathrm{~s}, 3 \mathrm{H}), 5.02(\mathrm{~s}, 2 \mathrm{H}), 7.06(\mathrm{~d}$, $2 \mathrm{H}, J=7.8 \mathrm{~Hz}), 7.13(\mathrm{~d}, 1 \mathrm{H}, J=1.2 \mathrm{~Hz}), 7.29-7.36(\mathrm{~m}, 3 \mathrm{H}) .{ }^{13} \mathrm{C}-\mathrm{NMR}(127$ $\mathrm{MHz}) \delta: 13.05\left(\mathrm{CH}_{3}\right), 50.15\left(\mathrm{CH}_{2}\right), 119.67\left(\mathrm{C},{ }^{3} J_{\mathrm{C}-\mathrm{F}}=4 \mathrm{~Hz}\right), 121.69\left(\mathrm{CF}_{3}\right.$, $\left.{ }^{1} J_{\mathrm{C}-\mathrm{F}}=267 \mathrm{~Hz}\right), 126.84(\mathrm{CH}), 128.40(\mathrm{CH}), 129.13(\mathrm{CH}), 130.46(\mathrm{C}$, $\left.{ }^{2} J_{\mathrm{C}-\mathrm{F}}=38 \mathrm{~Hz}\right), 134.88(\mathrm{C}), 146.39$ (C). MS $\mathrm{m} / z: 240\left(\mathrm{M}^{+}, 32 \%\right), 91(100 \%)$. Anal. Calcd for $\mathrm{C}_{12} \mathrm{H}_{11} \mathrm{~F}_{3} \mathrm{~N}_{2}$ : C, 60.00; H, 4.62; N, 11.66. Found: C, 60.02; $\mathrm{H}, 4.53 ; \mathrm{N}, 11.50$

1-Methyl-4-pentafluoroethyl-2-phenylimidazole $(\mathbf{4 h}): 4 \mathbf{h} ; \mathrm{mp} 56-57^{\circ} \mathrm{C}$ (hexane). ${ }^{1} \mathrm{H}-\mathrm{NMR}(500 \mathrm{MHz}) \delta: 3.76(\mathrm{~s}, 3 \mathrm{H}), 7.32$ (br s, $\left.1 \mathrm{H}\right), 7.44-7.46$ $(\mathrm{m}, 3 \mathrm{H}), 7.58-7.64(\mathrm{~m}, 2 \mathrm{H}) .{ }^{13} \mathrm{C}-\mathrm{NMR}(127 \mathrm{MHz}) \delta: 34.85\left(\mathrm{CH}_{3}\right), 111.00$ $\left(\mathrm{CF}_{2},{ }^{1} J_{\mathrm{C}-\mathrm{F}}=249 \mathrm{~Hz},{ }^{2} J_{\mathrm{C}-\mathrm{F}}=39 \mathrm{~Hz}\right), 119.12\left(\mathrm{CF}_{3},{ }^{1} J_{\mathrm{C}-\mathrm{F}}=286 \mathrm{~Hz},{ }^{2} J_{\mathrm{C}-\mathrm{F}}=39\right.$ $\mathrm{Hz}), 123.15(\mathrm{CH}), 128.70(\mathrm{CH}), 129.04(\mathrm{CH}), 129.27(\mathrm{C}), 129.56(\mathrm{CH})$, $130.00\left(\mathrm{C},{ }^{2} J_{\mathrm{C}-\mathrm{F}}=39 \mathrm{~Hz}\right), 149.36(\mathrm{C}) . \mathrm{MS} m / z: 276\left(\mathrm{M}^{+}, 100 \%\right)$. Anal. Calcd for $\mathrm{C}_{12} \mathrm{H}_{9} \mathrm{~F}_{5} \mathrm{~N}_{2}$ : C, 52.18; H, 3.28; N, 10.14. Found: C, 52.33; H, 3.32; N, 10.25 .

4-Heptafluoropropyl-1-methyl-2-phenylimidazole (4i): $4 \mathbf{i} ; \mathrm{mp} 49-50{ }^{\circ} \mathrm{C}$ (hexane). ${ }^{1} \mathrm{H}-\mathrm{NMR}(500 \mathrm{MHz}) \delta: 3.77$ (s, 3H), 7.32 (br s, 1H), 7.44-7.49 $(\mathrm{m}, 3 \mathrm{H}), 7.59-7.63(\mathrm{~m}, 2 \mathrm{H}) .{ }^{13} \mathrm{C}-\mathrm{NMR}(127 \mathrm{MHz}) \delta: 34.89\left(\mathrm{CH}_{3}\right), 101-$ $121\left(\mathrm{CF}_{2} \mathrm{CF}_{2} \mathrm{CF}_{3}\right), 123.41(\mathrm{CH}), 128.70(\mathrm{CH}), 129.05(\mathrm{CH}), 129.28(\mathrm{C})$, $129.56(\mathrm{CH}), 130.11\left(\mathrm{C},{ }^{2} J_{\mathrm{C}-\mathrm{F}}=39 \mathrm{~Hz}\right), 149.34(\mathrm{C}) . \mathrm{MS} \mathrm{m} / z: 326\left(\mathrm{M}^{+}\right.$, 78\%), 207 (100\%). Anal. Calcd for $\mathrm{C}_{13} \mathrm{H}_{9} \mathrm{~F}_{7} \mathrm{~N}_{2}: \mathrm{C}, 47.86 ; \mathrm{H}, 2.78 ; \mathrm{N}, 8.59$. Found: $\mathrm{C}, 47.65 ; \mathrm{H}, 2.87 ; \mathrm{N}, 8.68$

\section{References and Notes}

1) Resnati G., Tetrahedron, 49, 9385-9445 (1993); Banks R. E., Smart B. E., Tatlow J. C. (ed.), "Organofluorine Chemistry: Principles and Commercial Applications," Plenum, New York, 1994; Filler R., Kobayashi Y., Yagupolskii L. M. (ed.), "Organofluorine Compounds in Medicinal Chemistry and Biomedical Applications," Elsevier, Amsterdam, 1993; Hudlicky M., Pavlath A. E. (ed.), "Chemistry of Organic Fluorine Compounds II," ACS Monograph 187, American Chemical Society, Washington D.C., 1995; Ojima I., McCarthy J. R., Welch J. T. (ed.), "Biomedical Frontiers of Fluorine Chemistry," ACS Symposium Series 639, American Chemical Society, Washington D.C., 1996.

2) McClinton M. A., McClinton D. A., Tetrahedron, 48, 6555-6666 (1992); Kiselyov A. S., Strekowski L., Org. Prep. Proced. Int., 28, 289-318 (1996).

3) Grimmett M. R., "Advances in Heterocyclic Chemistry," Vol. 27, ed. by Katritzky A. R., Academic Press, New York, 1970, pp. 241-326; idem, "Comprehensive Heterocyclic Chemistry," Vol. 5, ed. by Potts
K. T., Pergamon, Oxford, 1984, pp. 345-498.

4) Jouneau S., Bazureau J. P., Tetrahedron Lett., 40, 8097-8098 (1999) and references cited therein

5) Baldwin J. J., Kasinger P. A., Novello F. C., Sprague J. M., J. Med. Chem., 18, 895-900 (1975); Scriabine A., Ludden C. T., Morgan G., Baldwin J. J., Experientia, 35, 1634-1637 (1979); Scriabine A., Ludden C. T., Watson L. S., Stavorski J. M., Morgan G., Baldwin J. J., ibid., 35, 653-655 (1979); Moazzam M., Parrick J., Indian J. Chem., 27B, 1051-1053 (1988); Carini D. J., Chiu A. T., Wong P. C., Johnson A. L., Wexler R. R., Timmermans P. B. M. W. M., Bioorg. Med. Chem. Lett., 3, 895-898 (1993); Brackeen M., Stafford J. A., Feldman P. L., Karanewsky D. S., Tetrahedron Lett., 35, 1635-1638 (1994); Elguero J., Fruchier A., Jagerovic N., Werner A., Org. Prep. Proced. Int., 27, 33-74 (1995); Li H.-Y., DeLucca I., Drummond S., Boswell G. A., J. Org. Chem., 62, 2550-2554 (1997); Hayakawa Y., Kimoto H., Cohen L. A., Kirk K. L., J. Org. Chem., 63, 9448-9454 (1998); Kamitori Y., Heterocycles, 53, 107-113 (2000).

6) Kawase M., Miyamae H., Kurihara T., Chem. Pharm. Bull., 46, 749756 (1998); Kawase M., Hirabayashi M., Saito S., "Recent Research Developments in Organic Chemistry," Vol. 4, Transworld Research Network, India, 2000, pp. 283-293.

7) a) Kawase M., J. Chem. Soc., Chem. Commun., 1994, 2101-2102; b) Kawase M., Saito S., Kurihara T., Heterocycles, 41, 1617-1620 (1995); c) Kawase M., Koiwai H., Yamano A., Miyamae H., Tetrahedron Lett., 39, 663 - 666 (1998); d) Kawase M., Koiwai H., Saito S., Kurihara T,. ibid., 39, 6189-6190 (1998); e) Kawase M., Miyamae H., Saito S., Heterocycles, 50, 71-74 (1999); f) Kawase M., Saito S., Chem. Pharm. Bull., 48, 410- 414 (2000).

8) a) Singh G., Singh S., Tetrahedron Lett., 1964, 3789-3792; b) Greco C. V., Gray R. P., Grosso V. G., J. Org. Chem., 32, 4101-4103 (1967).

9) Thompson L. A., Ellman J. A., Chem. Rev., 96, 555-600 (1996); Armstrong R. W., Combs A. P., Tempest P. A., Brown S. D., Keating T. A., Acc. Chem. Rev., 29, 123-131 (1996); Kobayashi S., Chem. Soc. Rev., 28, 1-15 (1999).

10) Booth R. J., Hodges J. C., Acc. Chem. Res., 32, 18-26 (1999); Guillier F., Orain D., Bradley M., Chem. Rev., 100, 2091-2157 (2000).

11) a) Mjalli A. M. M., Sarshar S., Baiga T. J., Tetrahedron Lett., 37, 2943-2946 (1996); b) Strocker A. M., Keating T. A., Tempest P. A., Armstrong R. W., ibid., 37, 1149-1152 (1996).

12) Bilodeau M. T., Cunningham A. M., J. Org. Chem., 63, 2800-2801 (1998).

13) Hamper B. C., Jerome K. D., Yalamanchili G., Walker D. M., Chott R. C., Mischke D. A., Biotech. Bioeng., 71, 28-37 (2000).

14) Kamitori Y., Hojo M., Masuda R., Wada M., Takahashi T., Heterocycles, 37, 153-156 (1994).

15) Baldwin J. J., Novello F. C., U.S. Pat., 4125530; [Chem. Abstr., 90, P121593n (1979)]

16) Deruiter J., Swearingen B. E., Wandreker V., Mayfield C. A., J. Med. Chem., 34, 2120-2126 (1991). 